\title{
Turbulent channel flow: comparison of streamwise velocity data from experiments and direct numerical simulation
}

\author{
J. P. MONTY† AND M. S. CHONG \\ Department of Mechanical Engineering, The University of Melbourne, Victoria 3010, Australia
}

(Received 25 June 2008 and in revised form 19 April 2009)

Recently there has been remarkable progress made in the direct numerical simulation (DNS) of wall-bounded turbulence, particularly of turbulent channel flow, with numerical data now available above $R e_{\tau} \approx 2000$ (Hoyas \& Jiménez, Phys. Fluids, vol. 18, 2006, p. 011702; Iwamoto et al., Proceedings of the Sixth Symposium Smart Control of Turbulence, 2005). Much knowledge has been gained from these results, particularly in the areas of flow structure and dynamics. Yet, while the value of such simulations is undoubted, only very limited comparisons with experimental data have been documented. Although the physics of the flow are captured correctly in an ideal DNS, as with any real numerical or physical experiment, there are opportunities for misrepresentation of the characteristics of turbulence. As such, this article seeks to make a comparison between a well-documented high Reynolds number $\left(R e_{\tau}=934\right)$, large box size $(8 \pi h \times 2 h \times 3 \pi h)$ DNS from del Álamo et al. (J. Fluid Mech., vol. 500, 2004, p. 135) and laboratory channel flow data measured by the authors. Results show that there is excellent agreement between the streamwise velocity statistics of the two data sets. The spectra are also very similar, however, throughout the logarithmic region the secondary peak in energy is clearly reduced in the DNS results. Although the source of the difference is not certain, the wavelengths concerned are close to the DNS box length, leading to the recommendation that longer box lengths should be investigated. Another large-scale spectral discrepancy near the wall results from the incorrect assumption of a constant convection velocity used to infer spatial information from the temporal. A near-wall convection velocity modification function is tentatively proposed. While the modification gives good agreement between the data sets, higher Reynolds number comparisons are required to better understand the intricate convection velocity issue.

\section{Introduction}

Any ingenuous wall-turbulence researcher will admit that progress in understanding this complex flow has been frustratingly slow over the past century. Indeed, there are very few statistical quantities or physical mechanisms of turbulence in pipes, channels or boundary layers whose behaviour is universally accepted. There are some, such as the spanwise scaling of the near-wall vortical structure (Kline et al. 1967), the mean velocity scaling in the viscous buffer region (Nickels \& Marusic 2001) and the occurance of the peak production of energy in the same region (Klebanoff 1954); but 


\begin{tabular}{lrrrrrrrr}
\multicolumn{1}{c}{ Source } & \multicolumn{1}{c}{$R e_{\tau}$} & $L_{x}$ & \multicolumn{1}{c}{$L_{z}$} & $\Delta x^{+}$ & $\Delta z^{+}$ & $N_{x}$ & \multicolumn{1}{c}{$N_{z}$} & \multicolumn{1}{c}{$N_{y}$} \\
Iwamoto et al. (2004) & 1160 & $6 \pi h$ & $2 \pi h$ & 12.6 & 4.7 & 1728 & 1536 & 769 \\
del Álamo et al. (2004) & 934 & $8 \pi h$ & $3 \pi h$ & 7.6 & 3.8 & 3072 & 2304 & 385 \\
Iwamoto et al. (2005) & 2320 & $6 \pi h$ & $2 \pi h$ & 12.6 & 4.7 & 3456 & 3072 & 1537 \\
Hoyas \& Jimenez (2006) & 2003 & $8 \pi h$ & $3 \pi h$ & 8.2 & 4.1 & 6144 & 4608 & 633
\end{tabular}

TABLE 1. Details of high-Reynolds-number DNS data of note. $L_{x}, L_{z}$ are the length and width of the box computed; $\Delta x^{+}, \Delta z^{+}$are the grid spacing in the streamwise and spanwise directions; $N_{x}, N_{y}, N_{z}$ are the number of collocation points in each direction.

these leave us a long way from even a basic understanding of the entire flow field, which is needed in order to improve the engineering of vehicles and transport systems that society heavily depends upon at this point in time. There may be many reasons for this lack of progress (a lack of high-Reynolds-number facilities is an important one, as discussed by Monty \& Chong 2007; Talamelli et al. 2007); however, the hindrances of instrumentation inadequacy or uncertainty and variations in boundary conditions between experiments in different facilities are two serious issues that are yet to be resolved. It is in these areas where the turbulence community stands to benefit most from numerical simulations as discussed by Jiménez (2003).

Numerical simulators interested in wall-turbulence commonly choose to simulate channel flow due to the statistical streamwise homogeneity (as opposed to external boundary layers) and absence of the cross-sectional grid problems associated with circular pipes. The first DNS of fully turbulent channel flow was documented in this journal by Kim, Moin \& Moser (1987); having a Kármán number of $R e_{\tau}=190$ $\left(R e_{\tau}=U_{\tau} h / v\right.$, where $U_{\tau}, h$ and $\nu$ are the friction velocity, channel half-height and kinematic viscosity, respectively), the data gave new insights into the behaviour of turbulence very near the wall despite the lack of scale separation. Over the two decades following the work of Kim et al. (1987), the progress in direct numerical simulations (DNS) of wall-bounded turbulence has been remarkable. Simulations up to $R e_{\tau}=2320$ with smooth and rough (or 'disturbed') walls were documented at the time of writing; four of note are detailed in table 1. Analyses of the numerical data have provided new insights into scaling laws (e.g. del Álamo et al. 2004; Hoyas \& Jiménez 2006) and the structural and dynamical characteristics of turbulence (e.g. del Álamo et al. 2006, Jiménez et al. 2004). The data has also been used by other researchers (e.g. Hutchins \& Marusic 2007a; Panton 2007) to make observations that are physically very difficult to achieve and, importantly, to guide future physical experiments at higher Reynolds number.

However, there have been few comparisons made between high-Reynolds-number DNS and physical data. This is not the fault of numerical researchers, but rather the lack of well resolved high-Reynolds-number measurements in a physical channel flow (notwithstanding the contributions of Zanoun, Durst \& Nagib (2003) and Monty 2005). Jiménez (2003) makes this point clear: 'Even today it is hard to find reliable laboratory experiments regarding the structural properties of turbulent channels beyond $R e_{\tau} \approx 1000$ '. Indeed, the only comparisons made by del Álamo et al. (2004), were with the pipe flow of Perry \& Abell (1977) and the boundary layer of DeGraaff $\&$ Eaton (2000). This will probably not concern the direct numerical simulator, since their simulations capture the physics of turbulence with accuracy limited only by the chosen parameters (e.g. box size, grid spacing, length and total number of time steps, etc.). Therefore, this article should not be considered a validation of the DNS data; 
it is a comparison designed to highlight the similarities, discrepancies and deficiencies of both.

The $R e_{\tau}=934$ DNS simulation by del Álamo et al. (2004) has been selected for comparison with the authors' laboratory measured data, which are presented for the first time here. Since the Reynolds number represents a ratio of small to large scales, it is imperative that sufficiently high Reynolds numbers are achieved in order that there is some separation between the scales, thus making the results useful to those investigating the higher $R e_{\tau}$ wall-turbulence commonly generated by real-world transport systems. There is evidence that $R e_{\tau} \approx 1000$ is in the range of a lower limit on Kármán number for this purpose (Hutchins \& Marusic $2007 b$, suggest $R e_{\tau} \approx 1700$ for a decade separation between dominant large and small scales in the log region), and so the conclusions drawn in this article will hopefully be of relevance as future higher Reynolds number DNS data become available.

In summary, it is the aim of this article to compare the mean velocity, turbulence intensity and one-dimensional energy spectra of a well-documented DNS and a new laboratory turbulent channel flow at 'high' Reynolds number.

\section{Experimental apparatus}

The channel flow facility at the University of Melbourne uses air as the working fluid and has a maximum bulk velocity, $U_{b(\max )} \approx 30 \mathrm{~m} \mathrm{~s}^{-1}$. The channel has half-height, $h=50 \mathrm{~mm}$ and width of $1170 \mathrm{~mm}$ so that the aspect ratio is $11.7: 1$, giving a central channel volume of nominally two-dimensional flow at least $600 \mathrm{~mm}$ (or 12h) in width (evidence for this claim is presented in Monty 2005). The distance from the 80-grit sand paper trip to the measurement point was $x=205 \times 2 h$, which was sufficient to produce fully developed flow, as shown in Monty (2005). Single point hot-wire measurements were made at 52 wall-normal locations with an automated traversing system (driven by a 400 pulse/revolution stepper motor and ball screw) with negligible backlash and capable of $5 \mu \mathrm{m}$ movements. The streamwise pressure gradient was used to determine friction velocity. A Kármán number of $R e_{\tau}=1040$ was achieved with a bulk velocity of $6.130 \mathrm{~m} \mathrm{~s}^{-1}, U_{\tau}=0.304 \mathrm{~m} \mathrm{~s}^{-1}$ and $v=1.477 \times 10^{5} \mathrm{~m}^{2} \mathrm{~s}^{-1}$. The Kármán number was slightly mismatched with the DNS, however, the $\sim 10 \%$ mismatch does not significantly affect the comparisons made, except very near the wall.

The hot-wire anemometer circuit used was a custom-made constant temperature type and gave a $50 \mathrm{kHz}$ frequency response to a step input. The hot-wire sensing element was made from wollaston wire with platinum core diameter of $2.5 \mu \mathrm{m}$ and etched length of $0.42 \mathrm{~mm} \pm 0.02 \mathrm{~mm}$, so that the non-dimensional wire length $l^{+}=8.5 \pm 0.4$. Ligrani \& Bradshaw (1987) suggests that this wire length is small enough to capture a high percentage of the small-scale energy at $y^{+}=15$ (the superscript ' + ' represents scaling with inner variables $U_{\tau}$ and $v$ ) for the Reynolds number studied; spatial resolution will be discussed in more detail in the following section. A $0.7 \mathrm{~mm}$ outer diameter Pitot-static probe was installed on the sting holding the hot-wire and adjusted to be equidistant from the wall when $y>0.35 \mathrm{~mm}\left(7.40 v / U_{\tau}\right)$. For hot-wire measurements below $y=0.35 \mathrm{~mm}$, the Pitot probe was allowed to press into the wall; no permanent deformation of the probe occurred through this procedure. The Pitot probe was placed a spanwise distance of approximately $25 \mathrm{~mm}$ from the hot-wire to ensure no interference between the probes. This set-up allowed initial and final calibration of the hot-wire at the centreline of the channel (thus eliminating movement of the hot-wire between calibration and measurement) where the shear is effectively zero across the Pitot diameter and the turbulence level is 

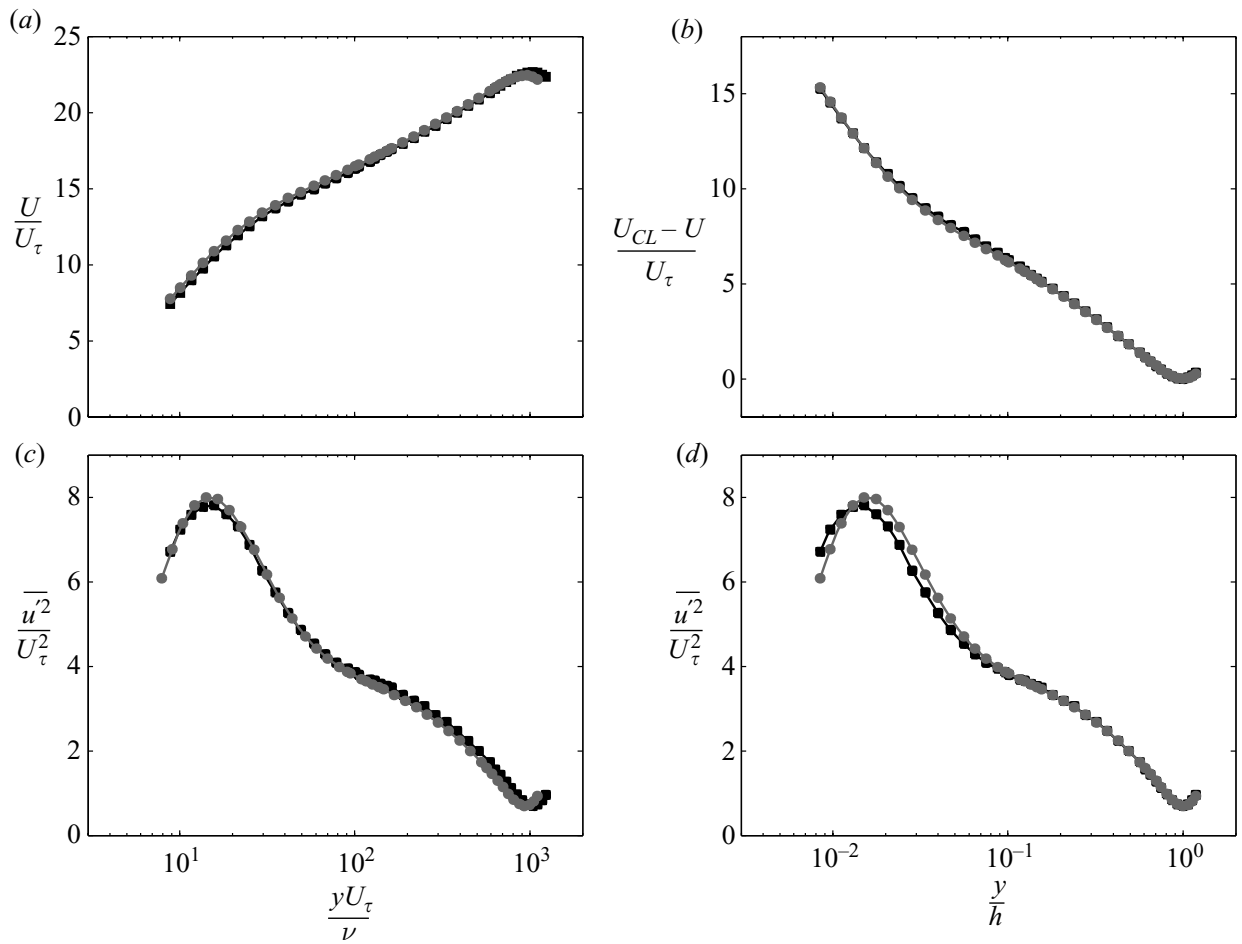

Figure 1. Comparison of the Melbourne channel (MC) with DNS •. Mean velocity with inner scaling $(a)$, and mean velocity defect with outer scaling $(b)$. Turbulence intensity: inner $(c)$ and outer $(d)$, scaling. Note that the DNS data has been interpolated onto the MC wall-normal coordinates for aesthetic improvement.

insignificant. Moreover, the two-probe set-up permitted a calibration check at each wall-normal location (for $y^{+}>7.4$ ), which is highly desirable when measuring for many hours without full recalibration. The experiment was repeated if there was significant disagreement $(>1 \%)$ between the results from each probe above $y^{+}=100$. All pressure measurements were made with an MKS Baratron 698A differential pressure transducer.

The analogue hot-wire circuit voltage was digitally sampled at $50 \mathrm{kHz}$ with a 14-bit Microstar Labs DAP4000a/212 data acquisition system for $10 \mathrm{~min}$ at each point. Both of these parameters are well above that required; e.g. 10 min represents a sampling time of over $73000 h / U_{b}$ or $81000 h / U_{C L}$, where $U_{b}, U_{C L}$ are the bulk and centreline velocities, respectively.

It is estimated that there is at most $1 \%$ error in hot-wire measured mean velocity and $2 \%$ in turbulence intensity. It should be noted that below $y^{+}=15(y / h=0.0144)$, the mean velocity drops below $2 \mathrm{~m} \mathrm{~s}^{-1}$, resulting in fluctuations below $0.5 \mathrm{~m} \mathrm{~s}^{-1}$. Calibration was only possible down to $\sim 0.7 \mathrm{~m} \mathrm{~s}^{-1}$ since the fan driving the flow cannot accurately maintain speeds lower than this and the error of the pressure transducer used is significant at such low pressure differences. For this reason, data below $y^{+} \approx 8$ were discarded and no comparisons are drawn from data below $y^{+}=15$.

\section{Streamwise velocity statistics}

Figure 1 shows the comparison of mean velocity and turbulence intensity. Note that the DNS data have been interpolated onto the more sparse wall-normal coordinates 
of the Melbourne channel (MC) data. Both inner and outer scaling is applied, with the outer scaling most important owing to the Reynolds number discrepancy. Clearly there is excellent agreement between the two data sets throughout the turbulent flow, particulary beyond $y / h=0.1$, where any Reynolds number effects are certainly absent. The most obvious differences are in the inner scaled mean velocity, where the DNS is up to $4 \%$ higher close to the wall (dropping to $\sim 1.5 \%$ in the log region). It is not clear why such a difference exists; the MC mean velocity has been compared to the DNS data of Spalart (1988) and agrees very well up to $y^{+}=100$ (Monty 2005). The turbulence intensity is also notably different near the wall, as seen in figure $1(c)$. At $y^{+}=15$ (peak turbulence intensity), for example, the MC measurement is approximately $3 \%$ lower than the DNS, despite its higher Reynolds number. This could be explained by the small, but not insignificant, small-scale energy contribution lost from the experiment due to the finite wire length $l^{+}=8.5$. The DNS data is arguably better resolved and, therefore, should exhibit a higher peak given that grid points are spanwise spaced by only $\Delta z^{+}=3.8$. However, an estimate of the expected differences due to spatial resolution cannot be provided, since the authors are not aware of studies into the quantitative effects of spanwise grid spacing on turbulence intensity in DNS.

Beyond the logarithmic region, $y / h \approx 0.1$, the agreement between the outer scaled data sets is remarkable, with maximum errors of $1 \%$ in mean velocity and turbulence intensity. However, it is reminded that the inner scaled velocities did not collapse as well, so that the outer scaled collapse infers a small $(\sim 1.5 \%)$ difference in the wakes of the two results.

\section{One-dimensional streamwise energy spectra}

The premultiplied power spectral density (PSD) of streamwise velocity fluctuations $k_{x} \Phi_{11}$, where $k_{x}$ is the streamwise wavenumber associated with the PSD. The premultiplied spectra are often referred to as energy spectra since, graphically, the area under a semi-log scale plot of this variable against the wavenumber is equal to the turbulence intensity. The energy spectra are plotted in figures $2-4$. All spectra will be plotted against wavelength $\lambda_{x}=2 \pi / k_{x}$. Although well-converged one-dimensional spectra were made publicly available by del Álamo et al. (2004), only a limited number of wall-normal locations were calculated by these authors. Many more wallnormal locations were required for the comparisons with the $\mathrm{MC}$ data and for the plot of figure 4. Therefore, the spectra of the DNS data were recalculated by the authors. To improve the spectral density of the DNS data, each plane was zeropadded to 32768 points long (this is effectively an interpolation of the data). Forty seven individual realizations of the entire DNS flow field were processed in an effort to improve convergence of the largest scales. However, it is clear that many more realizations are required to achieve better convergence as illustrated in figure 2 . This figure presents the authors' calculations in light grey circles and black solid lines. The light grey circles are the spectra averaged over all realizations, while the black lines are the same data with smoothing applied. The smoothing is a simple 15-point moving average type. The justification for this smoothing is given by comparison with the well-converged data of del Álamo et al. (2004), shown as dark grey squares in figure 2. It can be seen that the recalculated spectra are very close to the published data, with maximum differences occurring around $y / h=0.3$ and only at the largest wavelengths, where the better converged spectra are always lower in magnitude. Up to and including the logarithmic region and beyond $y / h=0.5$ all calculations are in acceptable agreement. While the differences should not be ignored, it will be evident 

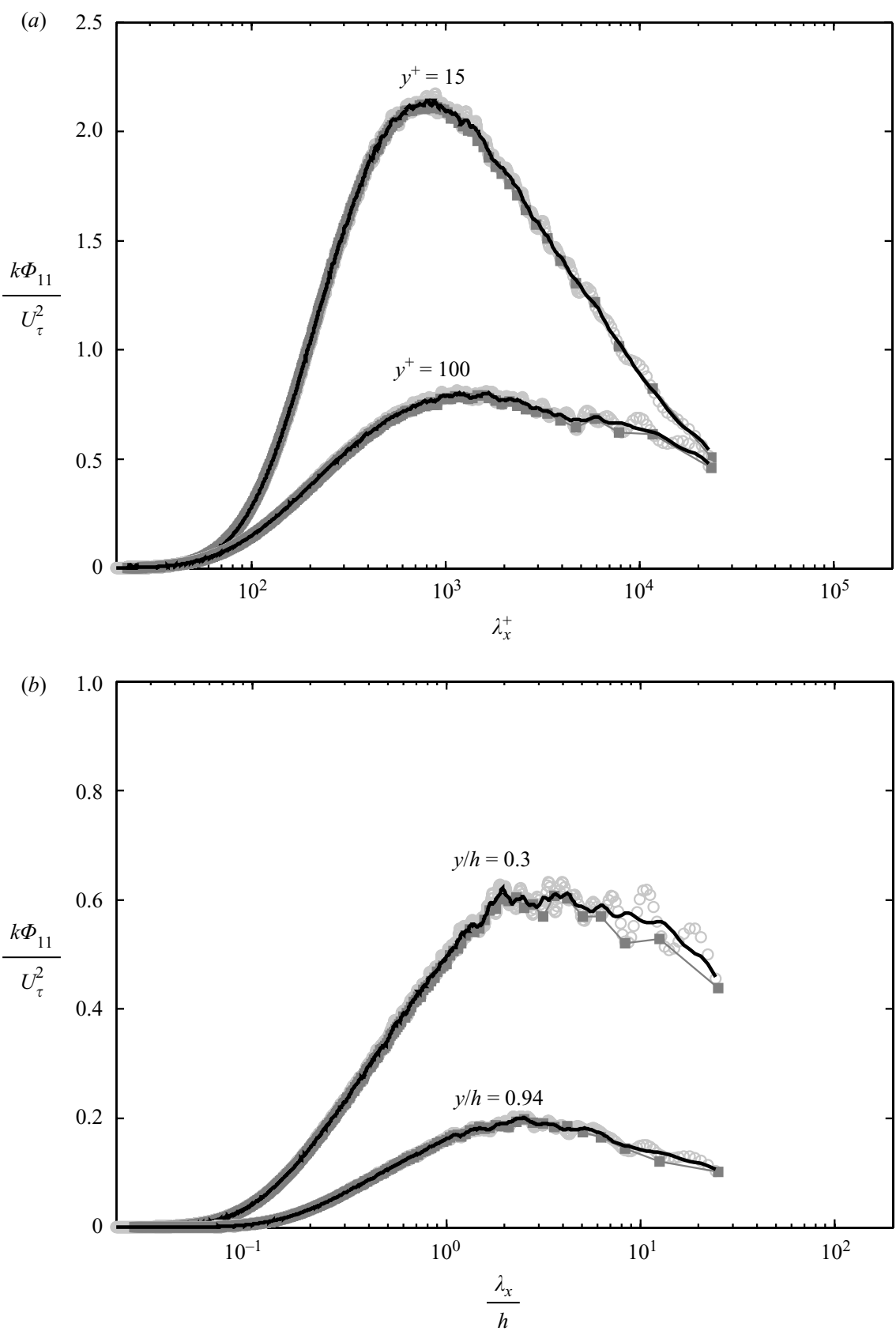

FIGURE 2. Comparison between premultiplied spectra from DNS data. Light grey circles: spectra averaged over 47 realizations of the DNS data (computed by the authors); black lines: averaged spectra with smoothing, which is the data used for all comparisons hereafter; dark grey squares: converged spectra computed by del Álamo et al. (2004).

in the following discussions that they do not affect the conclusions drawn from comparisons with the MC data.

The MC data, of which there are $30 \times 10^{6}$ samples at each wall-normal location, were split into windows of $2^{16}$ points $(1.31 \mathrm{~h} / U$, where $U$ is the local mean velocity) 


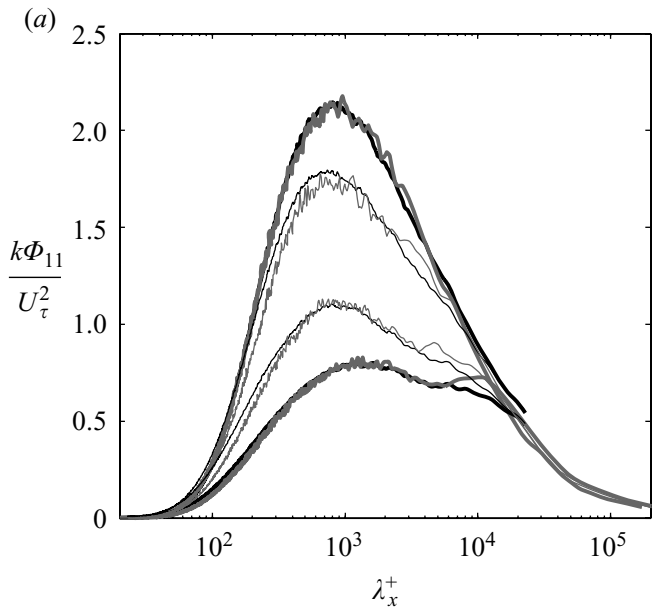

(b)

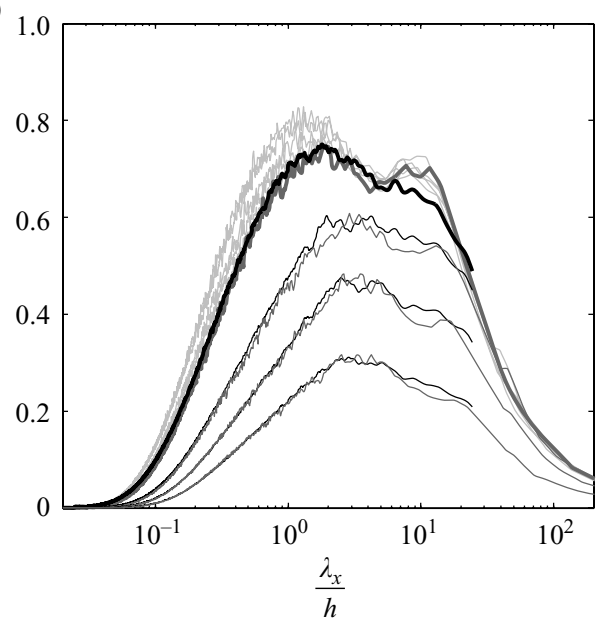

FIGURE 3. Premultiplied spectra at selected wall-normal locations. Black lines are DNS spectra; grey lines are MC spectra. Heavy lines are discussed in detail in the text. (a) from top to bottom: $y^{+}=15,24,50,100 ;(b)$ from top to bottom: $y / h=0.1-0.14$ (light grey, MC only), $\mathbf{0 . 1 5}, 0.32,0.5,0.7$. Emboldened values correspond to heavy lines.

to improve convergence of the small scales and zero padded to $2^{17}$ points to improve spectral resolution. It must be noted that the MC spectra are calculated from a time series and so require the invocation of Taylor's frozen turbulence hypothesis to infer the spatial spectrum from the temporal. For spectra presented in this section, a convection velocity equal to the local mean was chosen for this purpose.

The first comparison between the data sets involves the premultiplied spectra at selected wall-normal locations shown in figure 3. In this figure the wavelength is scaled with inner variables and data from $y^{+}=15-100$ are presented. The agreement is very good overall with both data sets showing very similar shaped spectral distributions; where differences are observed, they increase as the wall is approached due to the difference in $R e_{\tau}$ and the effect of wire length. In figure 3(b), the concentrated light grey lines represent all MC data in the logarithmic region. The collapse of the largescale secondary energy peak with outer scaling is clearly illustrated, with a peak energy of $k_{x} \Phi_{11} \approx 0.7 U_{\tau}^{2}$ at a wavelength $\lambda_{x} \approx 10 h$. Similar results have been reported in pipes and boundary layers by Guala, Hommema \& Adrian (2006) and Hutchins \& Marusic (2007a), although the secondary peak is at smaller wavelengths in boundary layers. del Álamo et al. (2004) also noted that the energy spectra collapse with outer variables in the vicinity of $\lambda_{x}=10 h$. At the edge of the $\log$ region $y / h=0.15$, both data sets are plotted with heavy lines for comparison and it is encouraging to see that the data are so similar for $\lambda_{x}<4 h$. However, the discrepancy seen at the beginning of the log region at large wavelengths remains, which is unsurprising given the scaling with outer variables. Interestingly, there appears to be an appreciable attenuation of energy in the DNS in the vicinity of $\lambda_{x}=10 h$. In fact, the secondary peak is rather less defined, resembling more of a shoulder. It could be suggested that the use of a constant convection velocity when applying Taylor's hypothesis could be the cause of this discrepancy. To reconcile the spectra in the log region, a modification to the convection velocity is required such that the largest structures $\left(\lambda_{x} \gtrsim 8\right)$ are convected significantly faster than the local mean. There are no obvious reasons for such an increased convection velocity given the current state of knowledge of the large-scale 

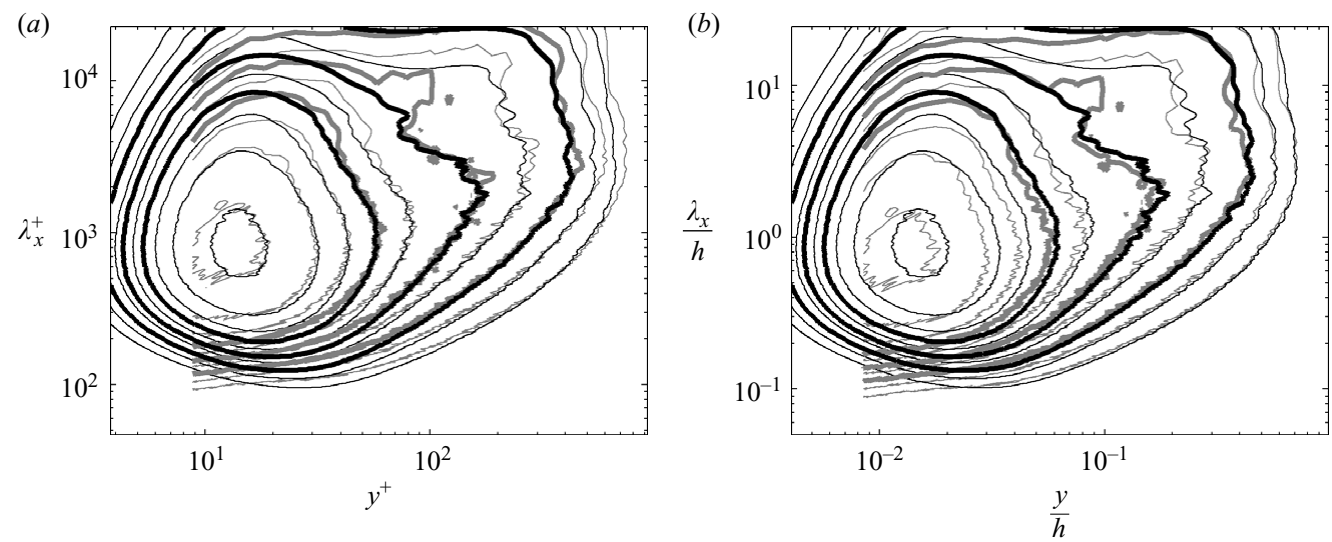

FIGURE 4. Spectra map presenting contours of $k_{x} \Phi_{11}$. Black lines are DNS data, grey lines are MC spectra. $k_{x} \Phi_{11} / U_{\tau}^{2}$ contour levels are, from outermost to innermost, $0.30,0.40,0.50, \mathbf{0 . 6 2}$, $0.72, \mathbf{0 . 8 5}, 1.00, \mathbf{1 . 2 0}, 1.50$ and 2.02. (a) Inner scaling; (b) outer scaling. Emboldened values correspond to heavy lines.

structure of wall turbulence. However, it is acknowledged that the proposition is physically feasible and a detailed convection velocity investigation could prove its validity (essentially extending the work of Dennis \& Nickels 2008).

Returning to figure $1(d)$, a difference of only $\sim 1 \%$ between turbulence intensities occurs in the logarithmic region and this is due to the missing secondary peak in the DNS. While $1 \%$ is an insignificant fraction of the average streamwise kinetic energy, the missing energy from the large scales remains a concern because Guala et al. (2006) found that structures of such high wavelengths (very large scale motions) are responsible for up to half of the Reynolds shear stress in the log region. Hutchins \& Marusic (2007b) and del Álamo \& Jiménez (2003) also show that these structures have a 'footprint' that reaches the wall, suggesting they are attached to the wall and are significant contributors throughout the flow. Since the wavelengths of the secondary peak and where the differences between the data sets are greatest (peaking at $\left.\lambda_{x} \approx 12-14 h\right)$ are so close to the box length of the DNS (25.13h), an insufficient box size could be the source of the problem. Conducting simulations with longer boxes would, therefore, provide clarification of the true nature of the spectral signature of the large-scales (along with the aforementioned convection velocity study).

Beyond the logarithmic region the two data sets again agree very well, even for large wavelengths. Recalling that the well-converged DNS spectra of del Álamo et al. (2004) display a little less energy at large wavelengths than the authors' calculations (as discussed above), it must be concluded that the simulation and the experiment are in agreement within experimental error. Interestingly, using the local mean as the convection velocity seems appropriate based on the evidence presented so far.

Recently, del Álamo et al. (2004) and Hutchins \& Marusic (2007a) have shown the plotting of 'spectra maps' to be a convenient way to present the extensive information contained in the wall-normal distribution of energy spectra. The 'spectra maps' are simply contours of premultiplied energy spectra, plotted with wavelength and wallnormal distance as the ordinate and abscissa. Such plots are provided in figure 4 with three contours highlighted for clarity, $k_{x} \Phi_{11} / U_{\tau}^{2}=0.5,0.72$ and 1.0. Inner and outer scaled maps are shown. Comparison of the two figures gives a very clear idea of the effect of the Reynolds number discrepancy between the data sets: the inner scaled 

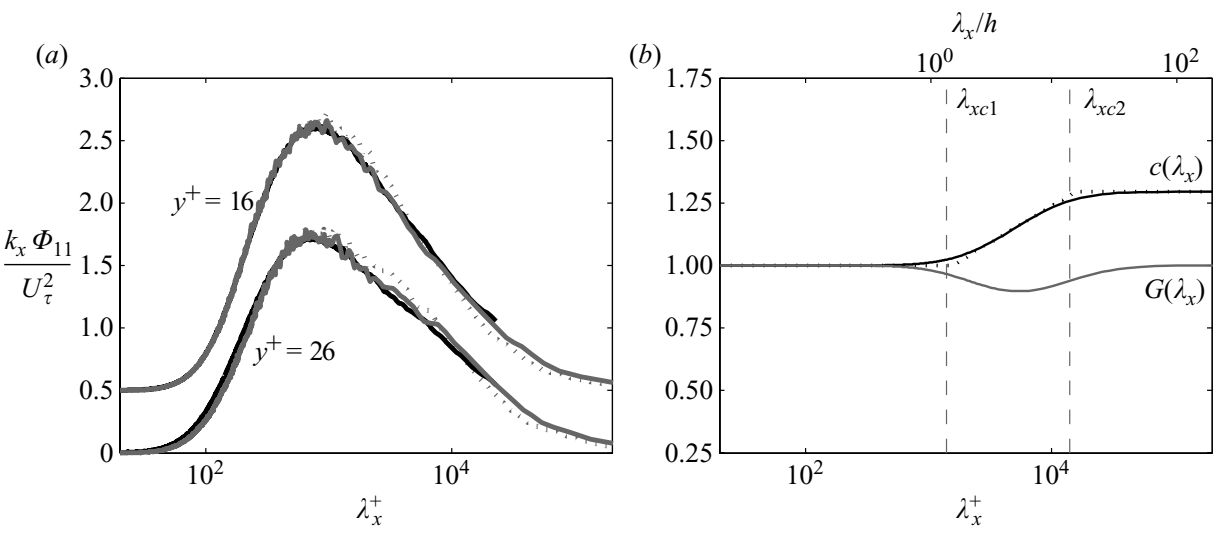

FIGURE 5. Results of modifying the convection velocity of a range of wavelengths. $(a)$ Comparison between: DNS, black; MC with constant convection velocity, dotted and; MC with convection velocity modification, heavy grey. (b) Example $\left(y^{+}=15\right)$ convection velocity modification functions: conceptual modification, dotted; actual modification function, $c\left(\lambda_{x}\right)$ and; energy redistribution function, $G\left(\lambda_{x}\right)$.

data display better agreement near the wall, while the outer scaled data are closer further from the wall.

Most importantly, figure 4 provides a global view of the energy distribution, which reveals information that is difficult to uncover from the single point comparisons of figure 3. First, the extent of the energy discrepancy around the secondary peak in the $\mathrm{MC}$ data is very clearly shown in this figure around $\left(y / h, \lambda_{x} / h\right)=(0.08,9)$. From examining one-dimensional plots of spectra and these spectra maps, it was found that the bounds of the large-scale energy difference roughly form a triangular region with vertices $\left(y / h, \lambda_{x} / h\right) \approx(0.1,6),(0.06,18),(0.2,18)$. Outside of this region the data are in excellent agreement except very close to the wall where $y \lesssim 0.05 \mathrm{~h} \approx 50 \mathrm{v} / U_{\tau}$. In this viscous region, Reynolds number and spatial resolution effects will be present in the MC data, yet these do not explain the extent of the trends seen here. At first sight, it appeared that the DNS channel flow has a stronger 'footprint' of largescale structure near the wall (more energy in the 12-14h wavelengths). As noted earlier, this 'footprint' has been clearly pointed out by Hutchins \& Marusic (2007a) from visualizations of the streamwise velocity fluctuations from this same DNS data. However, figure 3(a) (and perhaps more clearly in figure 5) shows that the decaying behaviour of the energy with increasing wavelength is quite different to the MC data for $\lambda_{x}>h$, not just around $\lambda_{x} \approx 12-14 h$. A close look at the $y^{+}=15$ spectra show that the two spectra decay roughly linearly in the region $3 h<\lambda_{x}<20 h$, but with different gradients. This results in the DNS having higher energy for $\lambda_{x}>4 h=4000 v / U_{\tau}$, despite its slightly lower $R e_{\tau}$. The only other reliable data at $R e_{\tau} \approx 1000$ that the authors were able to find in the literature, was the boundary layer spectra plotted in figure 2(a) of Hutchins \& Marusic (2007b). There the drop-off of large-scale energy has a very similar gradient to the MC data, although there is a bodily shift to lower wavelengths, as expected since the largest energetic scales in the boundary layer are shorter than in a channel or pipe. Whether this gradient should be the same as the channel flow is debatable, nonetheless, it is an interesting similarity. Further, it is difficult to imagine what could physically cause the large-scale energy to be stronger in the DNS when the large-scale structures are clearly weaker in the log region (where such wavelengths are most energetic). Considering these observations, the use of a 
constant convection velocity (the local mean) to infer the spatial wavelength from the temporal could explain the difference between experimental data and the DNS. Modifying the convection velocity as a function of wavelength is a complex exercise to which the following section is devoted.

Aside from the discussed discrepancies, figure 4 reiterates the remarkable agreement in streamwise energy spectra between the two data sets. This is particularly true in the outer flow region and for shorter wavelengths, where the two experiments are most likely to be mutually free from measurement or numerical inaccuracies.

\section{Convection velocity modification}

For the case of a constant convection velocity for all wavelengths, a global modification of this parameter is trivial. However, modifying the convection velocity for a range of wavelengths, so that the modification is a function of wavelength, is much more complex. The complexity arises due to the requirement of the energy spectrum to have an area equal to the streamwise turbulence intensity,

$$
\begin{aligned}
{\overline{u^{\prime 2}}}^{+}=\int \frac{k_{x} \Phi_{11}\left(k_{x}\right)}{U_{\tau}} \mathrm{d}\left(\log k_{x}\right) & =-\int \frac{k_{x} \Phi_{11}\left(\lambda_{x}\right)}{U_{\tau}^{2}} \mathrm{~d}\left(\log \lambda_{x}\right) \\
& =-\int \frac{k_{x} \Phi_{11}\left(\lambda_{x} c\left(\lambda_{x}\right)\right)}{U_{\tau}^{2}} \mathrm{~d}\left(\log \left[\lambda_{x} c\left(\lambda_{x}\right)\right]\right),
\end{aligned}
$$

where $c\left(\lambda_{x}\right)$ describes the convection velocity change for each wavelength (recall we start with the assumption that the convection velocity for all scales is the local mean $U_{l}$, so the modified convection velocity is $U_{c}=c U_{l}$ ). From (5.1), it can be seen that a convection velocity change cannot be implemented without a redistribution of the energy. This redistribution is easily determined from (5.1):

$$
\Phi_{11}\left(\lambda_{x} c\left(\lambda_{x}\right)\right)=\frac{c\left(\lambda_{x}\right)}{\lambda_{x} c^{\prime}\left(\lambda_{x}\right)+c\left(\lambda_{x}\right)} \Phi_{11}\left(\lambda_{x}\right)=G\left(\lambda_{x}\right) \Phi_{11}\left(\lambda_{x}\right),
$$

and its magnitude will be denoted as $G\left(\lambda_{x}\right)$. Note that $c^{\prime}$ is simply the derivative of $c\left(\lambda_{x}\right)$ with respect to the independent variable, $\lambda_{x}$. Now a form for the convection velocity modification function $c\left(\lambda_{x}\right)$ must be chosen. From figure 4 it is clear that in order to improve the agreement between the data sets, an increase in the convection velocity of the large scales is required. Due to the approximately logarithmically increasing velocity with wall distance (and assuming longer eddy structures are also taller), it seems logical to choose a logarithmically increasing convection velocity for some range of wavelengths, such as the dotted line shown in figure $5(b)$. In this figure the convection velocity is constant until a certain chosen wavelength, which should be characteristic of the largest structure that moves at the local mean velocity, then there is a logarithmic increase in convection velocity until another chosen wavelength, beyond which the structures are considered to have a different constant convection velocity (whether there should be a constant upper limit on convection velocity is debatable and will be briefly discussed later in this section). However, if the convection velocity change is not smooth, the energy redistribution function will be discontinuous because of the $c^{\prime}$ term and, therefore, so will the modified spectrum. Hence, the error function is used which is essentially a smoothed version of the conceptual logarithmic modification:

$$
c\left(\lambda_{x}\right)=\frac{M-1}{2} \operatorname{erf}\left(\frac{1}{B} \log \left(\lambda_{x}\right)-D\right)+\frac{M+1}{2}=\frac{U_{c}}{U_{l}},
$$


where $U_{c}$ is the modified convection velocity, $U_{l}$ is the local mean velocity, and it should be noted that $c$ will also be considered a function of wall-distance through $M=M(y)$, so that $c$ is a surface in $y-\lambda_{x}$ space. The chosen convection velocity modification curve at $y^{+}=15$ is shown in figure $5(b)$. The value of $M$ sets the final value of $U_{c}$ at a given wall-distance; that is, the convection velocity of the largest wavelengths. $B$ and $D$ determine the corner wavelengths, defined as the points where the dotted line of figure 5 begins and ends increasing. All constants are difficult to choose and curve-fitting is not preferred because: both independent $\left(\lambda_{x}\right)$ and dependent variables $\left(\Phi_{11}\right)$ are modified simultaneously; there is a small mismatch in Reynolds number and; some physical mechanism should be adhered to. The latter is most important and for that reason, the constants $B=1.27, D=1.23$ are chosen such that the corner wavelengths are $\lambda_{x c 1}=1.5 h$ and $\lambda_{x c 2}=15 h$. Thus, it is implied that the convection velocity should be fixed at: $i$. the local mean velocity for the wavelengths significantly less than $1.5 \mathrm{~h}$ ( $\sim 1500$ wall units) and; ii. a mean velocity equal to $M U_{l}$, where $U_{l}=U_{l}(y)$ is the local mean, for structures significantly longer than $15 h$, this length representing the longest energetic structures in the logarithmic region. The latter condition is most important as it recognizes the influence of the very large outer-scaled flow structures right down to the wall as discussed at length by Hutchins \& Marusic (2007a) and noted by del Álamo \& Jiménez (2003). All that remains, then, is to determine the value of $M(y)$, defining the convection velocity of the largest scales. It was initially thought that this should be the mean velocity of some point in the logarithmic region, say $M=U_{y^{+}=100} / U_{l}$. Recent preliminary work by $\mathrm{N}$. Hutchins (private communication) suggests 'superstructures' have a footprint at the wall that moves with a convection velocity close to the mean velocity at the midpoint of the $\log$ region, $U_{y^{+}=\sqrt{15 R e_{\tau}}}$, in high-Reynolds-number boundary layers. However, the best agreement with the DNS data was found by choosing a lower value, $M=U_{y^{+}=50} / U_{l}$. Furthermore, a closer look at figure 4 indicates that the differences in spectra at the large scales has mainly diminished by $y^{+}=50$, as discussed in the previous section. This is noteworthy because, if the convection velocity modification should end at $y^{+}=50$, the choice of $M=U_{y^{+}=50} / U_{l}$ produces a smooth decay of $c\left(\lambda_{x}\right)$ as $y^{+} \rightarrow 50$. However, it is reminded that the resolution of the large-scale structures in the DNS has been questioned, so the choice of $M$ should not be considered a truly appropriate one. Beyond $y^{+}=50, M(y)=1$, which is to say $U_{c}=U_{l}$.

It is most important to note that a number of other variations of the constants were tried (e.g. reducing and increasing $\lambda_{x c 1}$ and $\lambda_{x c 2}$ by factors of $2-4$, respectively) and gave very similar results to that shown, which raises a significant point: owing to the uncertainty and low Reynolds numbers of the data sets, the modified spectrum is somewhat insensitive to the choice of $M U_{l}$ (between the limits of $\sim U_{y^{+}=50}-$ $\left.U_{y^{+}=150}\right), \lambda_{x c 1}$ and $\lambda_{x c 2}$ suggested here. That is to say, physically plausible variations of these parameters produce cosmetic changes only; the general change to the shape of the spectra is the same and is in the desired direction. To determine the true nature of $c\left(\lambda_{x}\right)$ would require not only better matching of the parameters of the data sets (this is obvious), but more importantly, comparison at higher Reynolds number. As Reynolds number increases, the convection velocity of the largest scales and $\lambda_{x c 2}$ might be expected to change since the largest scales become more energetic (Hutchins \& Marusic 2007a), although the form of the convection velocity change (i.e. the logarithmic transition from local mean to characteristic log region velocity) should remain the same. It is also possible that the lower corner wavelength $\left(\lambda_{x c 1}\right)$, characterizing the wavelength at which the convection velocity begins increasing, 

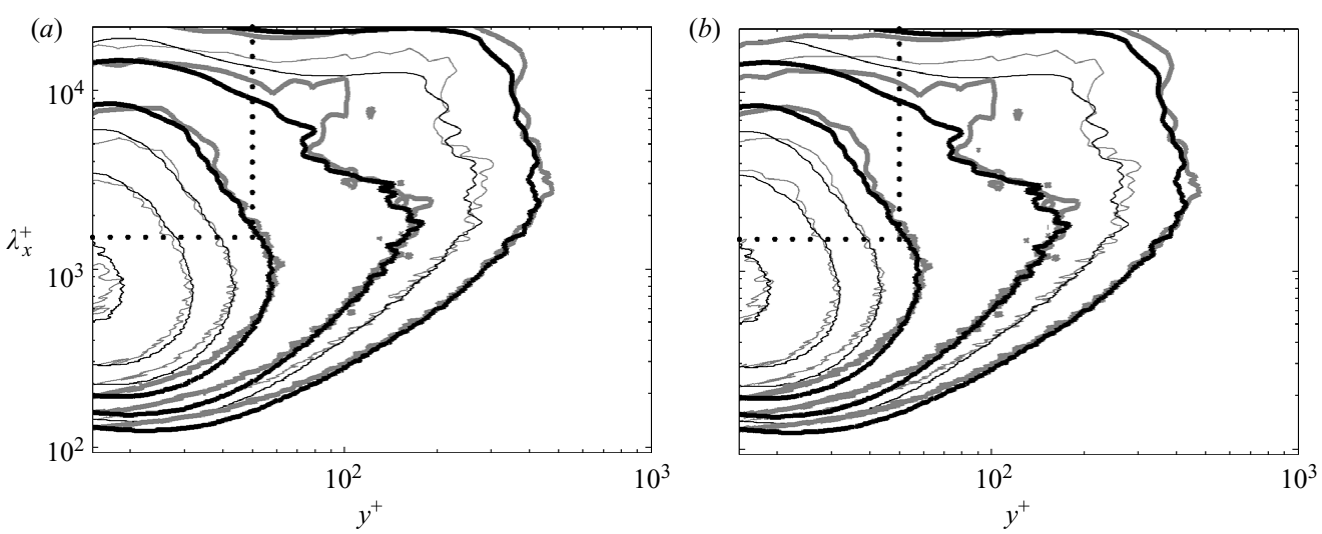

FIGURE 6. (a) Contour map of energy spectra showing results of modifying the convection velocity. DNS, black, and MC with convection velocity modification, grey. $k_{x} \Phi_{11} / U_{\tau}^{2}$ contours at levels of 0.50, 0.62, 0.72, 1.00, 1.20, 1.50 and 2.02 are shown. Emboldened values correspond to heavy lines. (b) Original contour map comparison shown in figure 4(a) where the local mean was used for the convection velocity. The dotted line highlights the region where the convection velocity modification is effective.

might be dependent on Reynolds number and so may be more appropriately specified in wall units, rather than channel half-heights.

The object of this discussion is not to provide a definitive form for the convection velocity, rather to show that a higher convection velocity is appropriate for larger eddies that have an influence all the way to the wall, and to provide a framework for future investigations at higher Reynolds number. Such investigations will almost certainly involve DNS data considering the extreme difficulty in acquiring accurate instantaneous measurements over a plane greater than $20 \mathrm{~h}$ long; just another indicator of the increasing importance of DNS data as computing power, and therefore, Reynolds number capability increase.

Whilst figure $5(a)$ gives a clear idea of the effect of modifying the convection velocity at two wall-normal locations, a more general view is provided in figure 6 . Here the contour maps of energy spectra are reproduced using the modified MC spectra (figure $6 a$ ) and compared with the previously shown MC spectra where the local mean was employed in Taylor's hypothesis (figure $6 b$ ). Inner scaling is used since only data below $y^{+}=50$ have been affected by the convection velocity change. Figure 6(a) contains the same DNS data shown in figures $4(a)$ and 6(b). The improved agreement with the $\mathrm{MC}$ energy distribution can be found in the region enclosed by the dotted lines, particularly at the largest wavelengths.

\section{Conclusions}

Comparisons between relatively high Reynolds number $\left(R e_{\tau} \gtrsim 1000\right)$ DNS and laboratory channel flows have shown excellent agreement in velocity statistics and in one-dimensional energy spectra. For data in the region $y^{+}>50$ and for wavelengths of $\lambda_{x}<4 h$, the two experiments agree well within experimental error. This gives the authors great confidence in their experimental facility and instrumentation. The comparison should also instill confidence in the numerical simulations, which have provided unprecedented access to the turbulent wall layer and have been analysed in a number of important investigations since their introduction. 
However, discrepancies were found. First, the large-scale energy behaviour of the experimental data very near the wall was found to deviate from the DNS. Since single-point hot-wire measurements require the use of Taylor's hypothesis to infer the spatial spectrum, it was shown that a modification of the convection velocity could improve the agreement between the data sets. The tentative modification proposed was essentially a logarithmic increase in velocity with wavelength, limited to the velocity at $y^{+}=50$, which was the point at which the large-scale energy differences were seemingly diminished. Much higher Reynolds number DNS $\left(\operatorname{Re}_{\tau} \mathcal{O}\left(10^{4}\right)\right.$, also with longer box length) and experimental data will be required to determine the true nature of the convection velocity over the full range of wavelengths. The most serious disagreement with the experimental data was an absence of the secondary peak in energy spectra for $\lambda_{x}>6 h$ in the DNS. The lost energy represents only a small percentage of the total streamwise turbulence intensity, yet it is a concern because these large-scale features have recently been identified as significant contributors to Reynolds shear stress and to have a strong 'footprint' near to the wall. It was acknowledged that an incorrect choice for the convection velocity for the large-scales in the log region could cause the energy discrepancy; a detailed investigation into convection velocity variations would, therefore, greatly benefit the understanding of the very large-scale structure. Even so, it is hoped that a result of this work will be the encouragement to increase the box length for DNS of turbulent channel flows in order to resolve any questions over the accuracy of the largest scales. Based on the Melbourne channel data, which has a secondary energetic peak in the log region at $8-12 h$ and a shoulder at $20 h$ even beyond $y / h=0.5$, a box length of at least $\sim 100 h$ would be required (an order of magnitude over the longest log region structures). According to Jiménez (2003) the total computational cost of the simulation increases with the square of box length, so that, at this point in time, the authors understand it is not a trivial matter to increase this parameter, even for $R e_{\tau}=1000$. However, at the current rate of increase in computational power available, this expense will not remain a limitation for long.

The authors are grateful for the financial support of the Australian Research Council through the Discovery Projects scheme (DP0556629). We also thank Professor R. Moser for allowing the use of DNS data.

\section{REFERENCES}

Del Álamo, J. C. \& Jiménez, J. 2003 Spectra of the very large anisotropic scales in turbulent channels. Phys. Fluids 15 (6), 41-44.

del Álamo, J. C., Jiménez, J., Zandonade, P. \& Moser, R. D. 2004 Scaling of the energy spectra of turbulent channels. J. Fluid Mech. 500, 135-144.

del Álamo, J. C., Jiménez, J., Zandonade, P. \& Moser, R. D. 2006 Self-similar vortex clusters in the turbulent logarithmic region. J. Fluid Mech. 561, 329-358.

DeGraaff, D. B. \& Eaton, J. K. 2000 Reynolds number scaling of the flat-plate turbulent boundary layer. J. Fluid Mech. 422, 319-346.

Dennis, D. J. C. \& Nickels, T. B. 2008 On the limitations of Taylor's hypothesis in constructing long structures in a turbulent boundary layer. J. Fluid Mech. 614, 197-206.

Guala, M., Hommema, S. E. \& Adrian, R. J. 2006 Large-scale and very-large-scale motions in turbulent pipe flow. J. Fluid Mech. 554, 521-542.

HoYAS, S. \& JimÉNEZ, J. 2006 Scaling of the velocity fluctuations in turbulent channels up to $R e_{\tau}=2003$. Phys. Fluids 18 (011702).

Hutchins, N. \& Marusic, I. 2007a Evidence of very long meandering features in the logarithmic region of turbulent boundary layers. J. Fluid Mech. 579, 1-28. 
Hutchins, N. \& Marusic, I. 2007b Large-scale influences in near-wall turbulence. Phil. Trans. R. Soc. A 365 (1852), 647-664.

Iwamoto, K., Fukagata, K., Kasagi, N. \& Suzuki, Y. 2004 DNS of turbulent channel flow at $r e_{\tau}=1160$ and evaluation of feedback control at practical reynolds numbers. In Proceedings of the Fifth Symposium Smart Control of Turbulence, February 29-March 2. Tokyo.

Iwamoto, K., Kasagi, N. \& SuzUKi, Y. 2005 Direct numerical simulation of turbulence channel flow at $r e_{\tau}=2320$. In Proceedings of the Sixth Symposium Smart Control of Turbulence, March 6-9. Tokyo.

JimÉNEZ, J. 2003 Computing high-Reynolds-number turbulence: will simulations ever replace experiments? J. Turbul. 4 (22), doi:10.1088/1468-5248/4/1/022.

Jiménez, J., Del Álamo, J. C. \& Flores, O. 2004 The large-scale dynamics of near-wall turbulence. J. Fluid Mech. 505, 179-199.

Kim, J., Moin, P. \& Moser, R. 1987 Turbulence statistics in fully developed channel flow at low Reynolds number. J. Fluid Mech. 177, 133-166.

KLEBANOFF, P. S. 1954 Characteristics of turbulence in a boundary layer with zero pressure gradient. Tech. Rep. TN3178. NACA.

Kline, S. J., Reynolds, W. C., Shrub, F. A. \& Rundstadler, P. W. 1967 The structure of turbulent boundary layers. J. Fluid Mech. 30, 741-773.

Ligrani, P. M. \& Bradshaw, S. 1987 Spatial resolution and measurement of turbulence in the viscous sublayer using subminiature hot-wire probes. Exp. Fluids 5, 407-417.

MonTy, J. P. 2005 Developments in smooth wall turbulent duct flows. PhD thesis, University of Melbourne, Victoria.

Monty, J. P. \& Chong, M. S. 2007 A review of recent investigations into high Reynolds number wall-turbulence. In Frontiers in Turbulence and Coherent Structures (ed. J. Denier \& J. S. Frederiksen), pp. 227-246. World Scientific.

Nickels, T. B. \& Marusic, I. 2001 On the different contributions of coherent structures to the spectra of a turbulent round jet and a turbulent boundary layer. J. Fluid Mech. 448, 367-385.

Panton, R. L. 2007 Composite asymptotic expansions and scaling wall turbulence. Phil. Trans. $R$. Soc. A 365 (1852), 733-755.

Perry, A. E. \& Abell, C. J. 1977 Asymptotic similarity of turbulence structures in smooth- and rough-walled pipes. J. Fluid Mech. 79, 785-799.

SpalaRt, P. R. 1988 Direct simulation of a turbulent boundary layer up to $\mathrm{R}_{\theta}=1410$. J. Fluid Mech. 187, 61-98.

Talamelli, A., Perisani, F., Fransson, J. J. M., Alfredsson, P. H., Johansson, A. V., Nagib, H. M., Ruedi, J. D., Sreenivasan, K. R. \& Monkewitz, P. A. 2009 Ciclope - a respone to the need for high Reynolds number experiments. Fluid Dyn. Res. 41, 021407.

Zanoun, E.-S., Durst, F. \& NAGIB, H. 2003 Evaluating the law of the wall in two-dimensionally fully developed turbulent channel flows. Phys. Fluids 15 (10), 3079-3089. 


\section{University Library}

\section{- M M I N E R VA A gateway to Melbourne's research publications}

Minerva Access is the Institutional Repository of The University of Melbourne

Author/s:

Monty, JP;Chong, MS

Title:

Turbulent channel flow: comparison of streamwise velocity data from experiments and direct numerical simulation

Date:

2009-08-25

\section{Citation:}

Monty, J. P. \& Chong, M. S. (2009). Turbulent channel flow: comparison of streamwise velocity data from experiments and direct numerical simulation. JOURNAL OF FLUID MECHANICS, 633, pp.461-474. https://doi.org/10.1017/S0022112009007769.

Publication Status:

Published

Persistent Link:

http://hdl.handle.net/11343/32994 